\title{
Hard X-ray Magnetic Tomography: A New Technique For The Visualization Of Three Dimensional Magnetic Structures
}

\author{
Claire Donnelly $^{1,2^{*}}$, Manuel Guizar-Sicairos ${ }^{2}$, Valerio Scagnoli ${ }^{1,2}$, Sebastian Gliga ${ }^{3}$, Mirko Holler ${ }^{2}$, Jörg \\ Raabe $^{2}$ and Laura J. Heyderman ${ }^{1,2}$. \\ 1. Laboratory for Mesoscopic Systems, Department of Materials, ETH Zurich, 8093 Zurich, Switzerland \\ 2. Paul Scherrer Institute, 5232 Villigen, Switzerland \\ 3. SUPA, School of Physics and Astronomy, University of Glasgow, Glasgow G12 8QQ, UK \\ * Corresponding author, claire.donnelly@psi.ch
}

Three-dimensional magnetic systems promise significant opportunities for technological applications, for example providing higher density data storage devices and new functionality associated with complex topology and greater degrees of freedom [1]. In particular, for three-dimensional magnetic structures, high-density three-dimensional magnetic data storage has been proposed [2]. In addition, curvature can lead to magnetochiral effects, which are not present in flat structures [3]. Within extended systems, such as permanent magnets and highly inductive magnetic materials that are widely used, for example, for energy harvesting and sensor technology, knowledge about the internal three-dimensional nature of the magnetization, including the details of the magnetic textures, is key for improving their performance. For the realization and optimization of these three-dimensional magnetic systems, however, an appropriate imaging technique is required.

Three-dimensional magnetic tomography of bulk structures was first achieved with neutron imaging [4], with which it is possible to determine the internal magnetic fields within an extended sample, with spatial resolutions down to tens of micrometres. Electron [5] and soft X-ray [6] tomography have also been demonstrated in 3D thin film structures with sub-10 nm spatial resolution. To bridge the gap between the high resolution obtained using soft x-rays and the ability to study bulk structures with $\mu \mathrm{m}$ resolution using neutrons, we have developed hard X-ray magnetic nanotomography, a high spatial resolution technique that takes advantage of the high penetration depth of hard X-rays, making possible the visualization of the internal three dimensional magnetic structure of an extended sample [7].

Beyond the measurement technique, one of the main challenges facing the investigation of threedimensional magnetic structures is the development of an algorithm for the tomographic reconstruction of the three-dimensional magnetization vector field. For vector tomography, rather than retrieving one scalar value for each voxel of the tomogram, one must obtain three independent components of the magnetization vector for each individual voxel. This has so far been achieved using available constraints, such as including prior knowledge of the magnetic properties of the sample [6,8]. In our work [7], we developed a new algorithm for the determination of the three dimensional magnetization vector field without requiring prior information of the properties of the sample, which we have validated with extensive numerical simulations [9].

We have combined this new algorithm with a dual axis tomographic setup to determine the internal magnetic configuration of a $\mathrm{GdCo}_{2}$ ferrimagnetic micropillar of $5 \mu \mathrm{m}$ diameter with a spatial resolution of $100 \mathrm{~nm}$. In this sample, the Gd and Co moments are antiparallel, with the material acting on average as a soft, low-moment ferromagnetic material with low magnetocrystalline anisotropy. To probe the magnetization within our sample, we make use of X-ray magnetic circular dichroism and measure 
tomographic projections with magnetic contrast employing ptychography [10], a coherent diffractive imaging technique which offers high sensitivity and high spatial resolution.

In Figure 2(a), the magnetic structure within the sample is obtained by considering the magnetization within an axial slice of the micropillar. As expected for a soft ferromagnetic material, the magnetization is smoothly varying, and one can identify a number of vortices, both clockwise and anticlockwise, as well as antivortices. When the three-dimensional volume magnetization of the pillar is considered, we can follow the evolution of the vortices through the height of the pillar, and observe that they intersect with magnetic domain walls. At the intersection points, magnetization singularities, known as Bloch points, occur, and we could resolve their surrounding structure, as shown in Figure 2(b,c). These singularities were first predicted over 50 years ago but were never directly observed until now [7].

This work represents a significant advance for magnetic imaging, and opens up the investigation of a wide variety of systems. While this first demonstration is based on ptychography, this method can be combined with a wide variety of microscopy techniques including scanning and full field X-ray microscopies. Moreover, with the advent of future generations of diffraction-limited synchrotron light sources and significant increases in coherent flux, the spatial resolution is expected to increase to the order of $20 \mathrm{~nm}$, drawing near the magnetic exchange length. Combination with soft X-rays could lead to sub-20 nm spatial resolution in the immediate future, albeit with thin film samples. In addition, we have recently extended our reconstruction algorithm to optimize simultaneously all projections from arbitrary sample orientations, leading to significant improvements in the reconstruction of magnetic structures [9].

References:

[1] A. Fernandez-Pacheco et al, Nat. Comm. 8, 15756 (2017)

[2] S. Parkin et al., Science 320, 190 (2008)

[3] R. Hertel, Spin 3, 134009 (2013)

[4] N. Kardjilov et al., Nat. Phys. 4, 399-403 (2008)

[5] C. Phatak et al., Nano Lett. 14, 759-764 (2014)

[6] R. Streubel et al., Nat. Commun. 6, 7612 (2015)

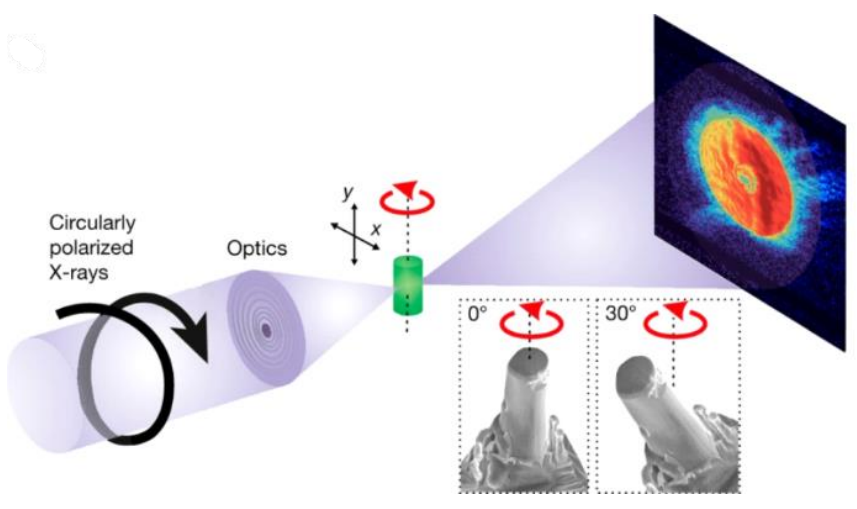

Figure. 1. Schematic of magnetic tomography setup. Ptychographic scans are performed with circularly polarized X-rays incident on the sample, which is rotated around the $y$-axis. To access all three components of the magnetization, tomographic data is collected for two sample orientations, as shown in the

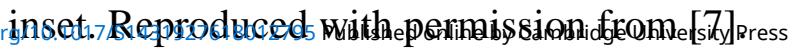

[7] C. Donnelly et al. Nature 547, 328 (2017),

[8] M. Suzuki et al., Appl. Phys. Express 11, 3 (2018).

[9] C. Donnelly et al., "Tomographic reconstruction of a $3 D$ magnetic vector field". In preparation.

[10] C. Donnelly et al., Phys Rev. B 94, 064421 (2016)

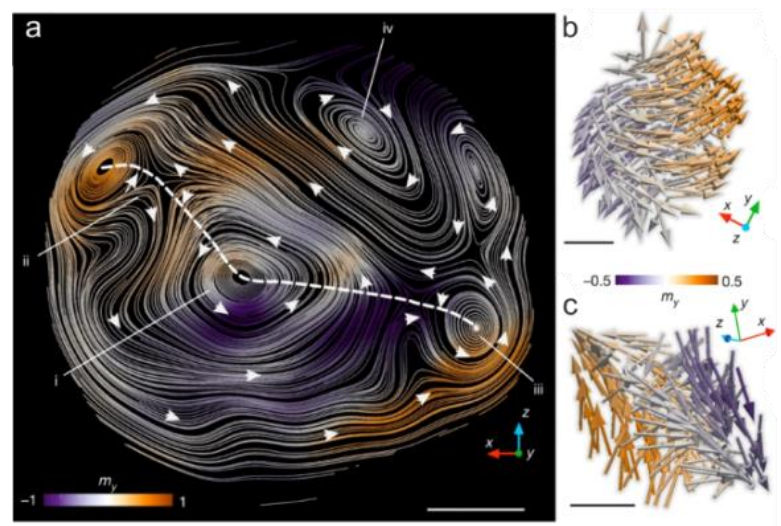

Figure. 2. The reconstructed magnetization of a) an axial slice and the magnetization surrounding $b$ ) a circulating Bloch point and c) an anti-Bloch point. Scale bars represent $1 \mathrm{um}$ in (a) and 100nm in $(b, c)$. Reproduced with permission from [7]. 